\title{
Review Article \\ Periodontal Disease and Age-Related Macular Degeneration: A Meta-Analysis of 112,240 Participants
}

\author{
Xuewen Lv $\mathbb{D}^{1,2}$ Weiqi Li $\mathbb{D}^{1,2}$ Zhiyu Fang $\mathbb{D}^{1,2}$ Xiaofei Xue $\mathbb{B}^{1,2}$ and Chunling Pan $\mathbb{D}^{1,2}$ \\ ${ }^{1}$ School and Hospital of Stomatology, China Medical University, Shenyang, China \\ ${ }^{2}$ Liaoning Province Key Laboratory of Oral Diseases, Shenyang, China
}

Correspondence should be addressed to Chunling Pan; chunlingpan@163.com

Received 19 May 2020; Revised 3 August 2020; Accepted 25 August 2020; Published 29 September 2020

Academic Editor: Alvin L. Young

Copyright (c) 2020 Xuewen Lv et al. This is an open access article distributed under the Creative Commons Attribution License, which permits unrestricted use, distribution, and reproduction in any medium, provided the original work is properly cited.

\begin{abstract}
Objective. Epidemiological studies have shown a correlation between periodontal disease (PD) and age-related macular degeneration (AMD). However, the results have been inconsistent, and no relevant meta-analysis has been performed on this topic. Hence, we performed a meta-analysis to evaluate whether the two diseases are related. Material and Methods. The PubMed, Embase, Cochrane Library, and Web of Science databases were searched up to April 20, 2020, for related articles. Two authors independently conducted literature screening and data extraction and then used the Stata 15.1 software to calculate the relative risk (RRs) and 95\% confidence intervals (CIs) to assess the association between PD and AMD. Results. A total of 5 observational studies involving 112,240 participants and 5,005 AMD patients were included. The results of meta-analysis using the random-effects model showed that the incidence of AMD in PD patients was 1.35 times that of non-PD patients; the difference was statistically significant $(\mathrm{RR}=1.35,95 \% \mathrm{CI}=1.07-1.70, P=0.011)$. Sensitivity analysis showed that the results were stable. Conclusions. PD patients have a higher risk of AMD, but the causal relationship between PD and AMD has not been confirmed. Further research should be carried out to verify the exact relationship between the two.
\end{abstract}

\section{Introduction}

Age-related macular degeneration (AMD) is an eye disease characterized by central visual impairment and can cause severe irreversible vision loss. It is an important cause of blindness and central blindness in people over 55 years of age [1]. In developed countries, AMD is the first cause of blindness [2]. AMD has two main types of macular degeneration, that is, dry AMD (nonexudative AMD) and wet AMD (exudative AMD). The main risk factors for AMD include age, smoking, family history and genetic factors, diet and nutritional factors, obesity, and cardiovascular disease (CVD) [3]. The pathogenesis of AMD is still unclear, and its formation mechanism may be the result of complex multifactor interactions between metabolic, functional, genetic, and environmental factors [4]. The treatment methods currently used are mainly for wet AMD, and there is no effective treatment for dry AMD, which accounts for the majority of clinical cases. The future research direction of treatment may be to prevent and regulate risk factors, so in-depth research on the risk factors for AMD is important [5].

Periodontal disease (PD) is an inflammatory and destructive disease that destroys the periodontal support tissue (gingival, periodontal ligament, alveolar bone, and cementum), leading to the formation of periodontal pockets, loss of attachment, and alveolar bone resorption. As the lesion progresses, teeth loosen, gums recede, and eventually tooth loss occurs [6]. The systemic promoters of periodontal disease include genetics, sex hormones, smoking, related systemic diseases (such as diabetes and immune dysfunction), and mental stress [7]. Many studies have shown that periodontal disease has a two-way relationship with systemic health or diseases and is an important risk factor for cardiovascular and CVD, diabetes, and respiratory diseases [8-12].

Although the exact pathogenesis of AMD is still unclear, the theory of inflammation and immunology is gaining more and more attention, and the role of various inflammatory factors and immune factors has been confirmed by experiments 
and histopathological studies [13-15]. One possible mechanism for PD to affect systemic diseases is that periodontal bacteria and their metabolites (such as endotoxin) enter the systemic circulation, and when they reach other parts of the body, they activate monocytes/macrophages and produce a large number of inflammatory factors, which in turn causes inflammation in other organs [16, 17]. Given that PD and AMD share some common risk factors, and PD may cause AMD's immune response and inflammation through bacteria and their products, Klein et al. [18] reported that the history of periodontal disease was related to the increase in retinal pigment. Since then, some other relevant epidemiological studies had been published, but these studies had provided inconsistent results [18-22]. There is no relevant metaanalysis to quantitatively evaluate the connection between the two. The purpose of this study is to systematically review existing literature and use meta-analysis to analyze whether $\mathrm{PD}$ and $\mathrm{AMD}$ are independently related.

\section{Materials and Methods}

This study was based on the statement of the Preferred Reporting Items for Systematic Reviews and Meta-Analyses (PRISMA) [23].

2.1. Eligibility Criteria. Studies that met the following conditions can be included: (1) the research theme was to explore whether PD is related to AMD; (2) observational studies, including case-control studies, cross-sectional studies, and cohort studies; (3) studies that provided raw data, or relative risk (RRs), odds ratios (ORs), or hazard ratios (HRs), and their 95\% CIs; (4) full-text articles were available and published in English, excluding letters to editors, meeting abstracts, case reports, and reviews; and (5) the study included a control group.

When different studies of the same population were included, we selected a longer follow-up period or extract data from more complete studies. The two authors (XWL and WQL) chose independently, and if there were differences, they resolved through discussion or consultation with the third author (CLP).

2.2. Search Strategy. A computerized search of the PubMed, Embase, Cochrane Library, and Web of Science databases was performed. We collected case-control studies, crosssectional studies, and cohort studies of the relationship between PD and AMD; the search time limit was from the establishment of the database to April 20, 2020. In addition, the references included in the literature are retrospectively included to supplement the relevant literature. The following keywords and thematic terms were used: "Periodontal Diseases", “Age-Related Macular Degeneration", “Drusen”, "Geographic Atrophy", "Retinal Pigment Epithelial Detachment", "Choroidal Neovascularization", and "Polypoidal choroidal vasculopathy". In Supplementary Materials, we have given specific electronic search criteria for each of the above databases (Appendix 1).

2.3. Data Extraction. The two authors (XWL and WQL) independently used standardized tables for data extraction, and disputes were resolved through discussion or consultation with the third author (CLP). Contents included the first author, published year, sample, study period, study design, follow-up period, age, AMD patients/participants, PD ascertainment, AMD ascertainment, AMD/age status, adjusted RRs (including ORs/HRs), and 95\% CIs, as well as adjustment factors and information required for quality assessment.

2.4. Quality Evaluation. Case-control studies and cohort studies used the Newcastle-Ottawa scale (NOS) for risk assessment of bias [24], and cross-sectional studies were evaluated by the bias risk evaluation criteria recommended by the Agency for Healthcare Research and Quality (AHRQ) [25]. The NOS scale includes a total of 8 items in three parts. The semiquantitative principle of the star system was used to evaluate the quality of the research. The full score is 9 stars; 0-3 stars, 4-6 stars, and 7-9 stars were defined as low, medium, and high quality. The standard recommended by AHRQ consists of 11 items, which were answered with "yes," "no," and "unclear," respectively. If the answer of an item was "no" or "unclear," the item's score was "0." If the answer was "yes," then the project score was " 1 "; 0-3 points, 4-7 points, and 8-11 points were defined as low, medium, and high quality. Disagreements were resolved through consensus between the two authors (XWL and WQL) or arbitration by the third author (CLP). When the study quality was defined as medium or high quality, the data in this study was included for meta-analysis.

2.5. Statistical Analysis. In observational studies, for smallprobability events, OR can be considered approximately equal to RR [26]. For cohort studies that report HR because of survival data, HR is approximately RR [27]. Some studies only give the respective effect sizes and their 95\% CIs for each severity of $\mathrm{PD}$ or AMD and did not give the total effect size and its 95\% CI representing the correlation between PD and AMD. In order to observe whether PD and AMD are related in general, we used the fixed-effects model to combine the data of different degrees of disease in the same study, so as to obtain the total effect size and 95\% CI of the study [28]. We converted the effect sizes and their 95\% CIs into their logarithms and standard errors and then merged them. A chisquared test was used to analyze the heterogeneity between the included studies. At the same time, $I^{2}$ was used to quantitatively judge the heterogeneity, and the inspection level was set to $\alpha=0.10$. When $P$ value $<0.1$ or $I^{2}>50 \%$, obvious heterogeneity was defined [29]. Usually, the random-effects model is used when there is obvious heterogeneity; otherwise, the fixed-effects model is used [29]. However, considering the obvious clinical heterogeneity caused by the different diagnostic criteria of PD and AMD and the methodological heterogeneity caused by the design and quality of studies, we decided to choose a random-effects model for statistical analysis. Subgroup analysis is based on study design, study areas, whether to adjust smoking, diabetes, hypertension, CVD, hepatitis B infection, study quality, and age status. Sensitivity analysis was to delete one study at a time and combined the remaining study data through a random-effects model to 
explore the degree of impact of the study on the total effect sizes and the robustness of the results. Since the number of studies was less than 10, no funnel plots were made. Begg's test and Egger's test were used to detect publication bias $[30,31]$. All statistical analyses were completed by the two authors independently using Stata version 15.1 and $\mathrm{R}$ version 4.0.0, and the $P$ values $<0.05$ were considered statistically significant. Disagreements were resolved through discussion by the two authors (XWL and WQL) or consultation with the third author (CLP).

\section{Results}

3.1. Study Selection and Characteristics. A total of 178 relevant literatures were obtained in the initial examination. After layer-by-layer screening, 5 studies were finally included [18-22], including 112,240 participants and 5,005 AMD patients. The search flow chart is shown in Figure 1. The included studies were published between 2008 and 2019 and included 4 cross-sectional studies and 1 retrospective cohort study. The characteristics of the studies are shown in Table 1. Five studies had adjusted the confounding factors, and Table 2 lists the detailed information. We evaluated the quality of all included studies. One item was classified as medium quality and four items as high quality (Tables 3 and 4).

\subsection{Results of Meta-analysis}

3.2.1. Overall Estimation. Meta-analysis results using the random-effects model showed that the incidence of AMD in PD patients was 1.35 times that of non-PD patients, and the difference was statistically significant $(R R=1.35,95 \% C I$ $=1.07-1.70, P=0.011)$. The heterogeneity test results found obvious heterogeneity $\left(I^{2}=80.4 \%, \quad P\right.$ for heterogeneity $<0.001)$. Detailed information is listed in Figure 2.

3.2.2. Sensitivity Analysis. Figure 3 lists the results of the sensitivity analysis. After excluding the included studies one by one, the results showed no substantial changes, suggesting that the meta-analysis results were robust. After excluding Shin et al.'s study [21], the combined RR $=1.56$ (95\% CI: 1.45-1.67, $I^{2}=0.0 \%$, heterogeneity for $\left.P=0.550\right)$. After removing the cohort study by Sun et al. [22], the combined $\mathrm{RR}=1.23$ (95\% CI: $1.00-1.51, I^{2}=42.1 \%$, heterogeneity for $P=0.159)$. The results did not change, and the heterogeneity decreased, indicating that these two studies contributed greatly to the statistical part of the overall meta-analysis heterogeneity.

3.2.3. Subgroup Analysis and Metaregression. Figure 4 lists the details of the subgroup analysis and metaregression. The RR values of all subgroups were $>1.00$. According to the study design, whether to adjust smoking, diabetes, CVD, and hepatitis B infection grouping, the heterogeneity decreased significantly. But metaregression found that the differences between the subgroups were statistically significant only in the subgroup of hepatitis B infection.
3.2.4. Publication Bias. According to the results of Egger's $(P=0.509)$ and Begg's test $(P=0.806)$, we found no obvious publication bias.

\section{Discussion}

There is no unified conclusion on whether PD and AMD are related, and the focus of each research result is different. Klein et al. [18] believed that the history of PD is related to the incidence of early AMD; Karesvuo et al. [19] confirmed that male alveolar bone loss is independently associated with AMD; Wagley et al. [20] found that PD is independently associated with AMD under 60 years of age or younger; Shin et al. [21] believe that in 62-year-old or younger people, only severe PD is independently related to AMD; cohort studies by Sun et al. [22] indicate that PD patients are more likely to develop AMD. And there is no relevant meta-analysis, so we think it is necessary to discuss whether PD and AMD are related in general. The meta-analysis results of the existing epidemiological evidence show that PD is associated with AMD, and the risk of AMD in PD patients is 1.35 times that of non-PD patients (95\% CI: 1.07-1.70, $P$ $=0.011) . I^{2}=80.4 \%, P$ for heterogeneity $<0.001$, and it can be considered obvious heterogeneity. Through subgroup analysis, we found that the type of study, whether to adjust smoking, diabetes, CVD, and hepatitis B infection reduced heterogeneity. Combined with the metaregression results, whether to adjust for hepatitis B infection may be the source of heterogeneity. Some studies have shown that hepatitis B infection is also a risk factor for AMD [32], and only a study by Shin et al. [21] adjusted this confounding factor; smoking, hypertension, and CVD are common risk factors for PD and AMD $[6,33]$. The results of the subgroup analysis show that the above three factors may cooperate with $\mathrm{PD}$ to increase the risk of $\mathrm{AMD}$; diabetes is also a common risk factor for PD and AMD [11,34], but our subgroup analysis results show that after adjusting for the confounding factor of diabetes, the correlation between PD and AMD was higher. We considered that there may be fewer studies included, and the results of Shin et al. may be the source of heterogeneity, but their study did not control the factor of diabetes, thus affecting the results of the diabetes subgroup. Subgroup analysis results according to the study quality show that the effect value did not change significantly, indicating that the overall research results are robust. Age is also a common and main risk factor for PD and AMD, and AMD mostly occurs in people over 50 years old. If the proportion of participants under 50 years old is too large, the prevalence of AMD may be underestimated in the original studies and then impact the accuracy of our results. There were 3 studies in the existing evidence that provided the effect sizes and 95\% CIs of the younger and elder age groups. After subgroup analysis and metaregression, we found that the correlation between $\mathrm{PD}$ and $\mathrm{AMD}$ in the younger group $\left(\mathrm{RR}=1.46,95 \% \mathrm{CI}: 1.22-1.74, I^{2}=35.2 \%\right)$ was stronger than the elder group ( $\mathrm{RR}=1.29,95 \% \mathrm{CI}: 0.81-2.16, I^{2}=93.4 \%$ ). We believed that this may be due to the following reasons: First, we thought that PD may have a closer relationship with the early stages of AMD. Second, although the age 


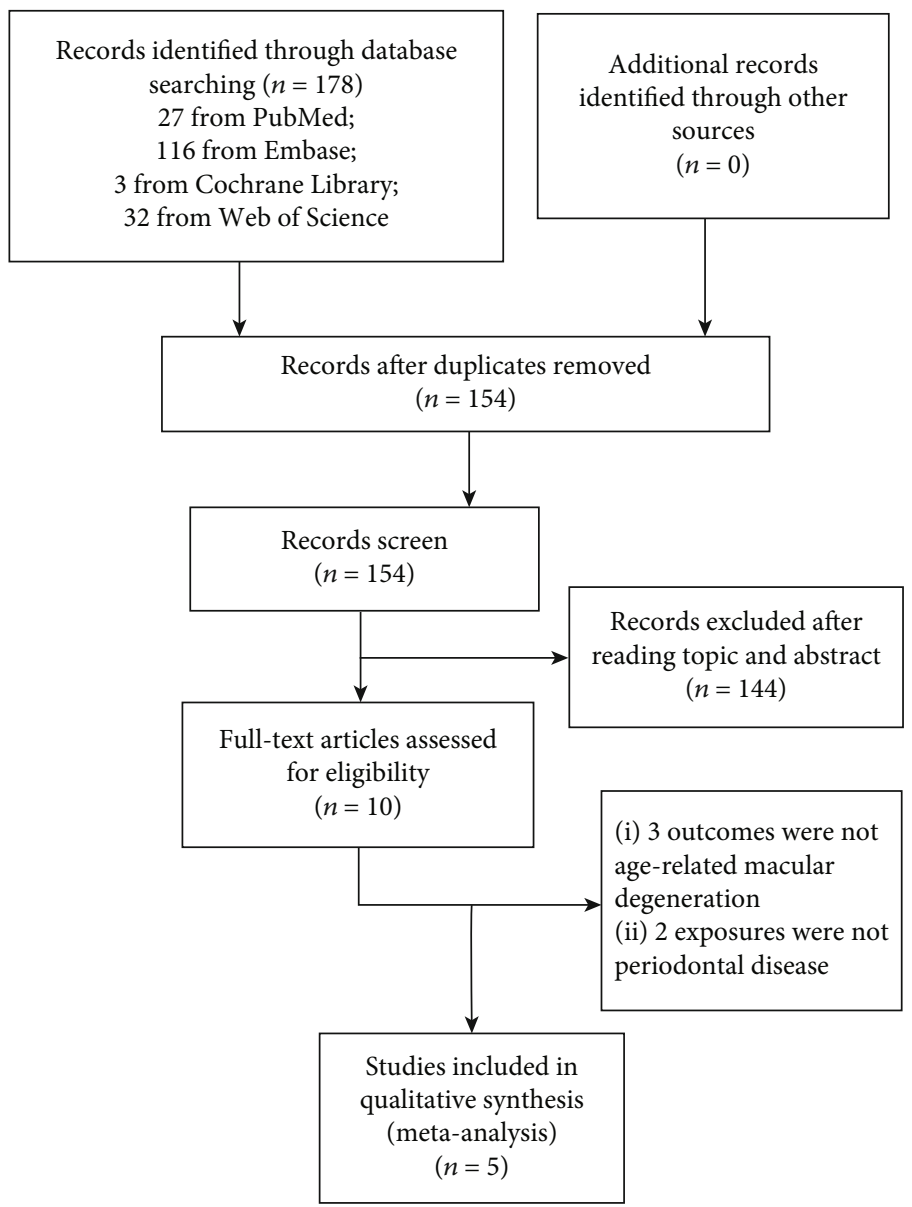

FIGURE 1: Search flow chart of the meta-analysis.

mix factor was adjusted, the effects of PD on AMD were diluted with other changes in the body as we age. Finally, we have only three included studies grouped according to the age group, impacting the reliability of our final results. However, the result of metaregression showed that the difference between the younger and elder age groups was not statistically significant, indicating that although the included studies included participants under the age of 50, we may have underestimated the prevalence of AMD, but it did not affect the final results. But due to the lack of original data, we could not adjust the age in the metaregression of each subgroup; these factors affected the reliability of metaregression in our study. After the sensitivity analysis excludes one study at a time, the meta-analysis results have not changed significantly. Egger's test and Begg's test did not find obvious bias.

In recent years, many studies have shown that periodontal infection may be a risk factor for cardiovascular disease, diabetes, pregnancy complications, respiratory infections, rheumatoid arthritis, and so on [8-12]. Periodontal bacteria and their products enter the systemic circulation, activate immune cells in other body parts to release proinflammatory cytokines, and form a chronic inflammatory state, which in turn leads to the development of some diseases, such as atherosclerosis [35] and premature delivery [36]. Studies have found oral pathogens in human atherosclerotic plaques [37], and Kalayoglu et al. [38] have found oral pathogens-Chlamydia pneumoniae - in human choroidal neovascular membranes excised from patients with wet AMD. We speculated that PD may play a role in the development of AMD through a mechanism similar to the above. The pathogenesis of AMD is unclear, but the theory of inflammation and immunology has received increasing attention from researchers [13-15]. Aging causes a decrease in the ability of retinal pigmented epithelium (RPE) cells to clear metabolic products, aggravating oxidative damage to the retina, and synergistic effects of metabolic, functional, genetic, and environmental risk factors, resulting in the accumulation of retinal toxic elements. In response to the increase in tissue damage, various immune pathways are inappropriately activated, including the classical and alternative complement pathways, the inflammasome, and Toll-like receptor (TLR) signaling. Eventually, the continuous activation of these proinflammatory and injury pathways leads to the development of advanced AMD and irreversible loss of vision [39]. Therefore, we have reason to believe that inflammation plays an important role in the relationship between PD and AMD. Periodontal infection may activate immune pathways on the retina through systemic circulation to promote the development of AMD. 
TABLE 1: Characteristics and quality assessment of the included studies.

\begin{tabular}{|c|c|c|c|c|c|c|c|c|c|}
\hline $\begin{array}{l}\text { First author, } \\
\text { published } \\
\text { year }\end{array}$ & $\begin{array}{c}\text { Sample } \\
\text { study period }\end{array}$ & Study design & $\begin{array}{l}\text { Follow- } \\
\text { up } \\
\text { period }\end{array}$ & $\begin{array}{l}\text { Age } \\
\text { (yrs) }\end{array}$ & $\begin{array}{c}\text { AMD } \\
\text { patients/participants }\end{array}$ & $\begin{array}{c}\mathrm{PD} \\
\text { ascertainment }\end{array}$ & $\begin{array}{c}\text { AMD } \\
\text { ascertainment }\end{array}$ & $\begin{array}{c}\text { AMD/age } \\
\text { status }\end{array}$ & $\begin{array}{c}\text { Risk } \\
\text { estimates } \\
(95 \% \mathrm{CI})\end{array}$ \\
\hline Klein, 2008 & $\begin{array}{l}\text { MESA; USA } \\
\text { 2002-2004 }\end{array}$ & $\begin{array}{l}\text { Cross- } \\
\text { sectional }\end{array}$ & 2 & $\begin{array}{l}45- \\
85\end{array}$ & $265 / 5887$ & $\begin{array}{c}\text { Detailed } \\
\text { questionnaire }\end{array}$ & $\begin{array}{c}\text { Ocular } \\
\text { fundus } \\
\text { photographs }\end{array}$ & $\begin{array}{l}\text { Early } \\
\text { Late } \\
\text { All }^{\#}\end{array}$ & $\begin{array}{c}\text { OR }=1.32 \\
(0.98- \\
1.79) \\
1.49(0.63- \\
3.50) \\
1.34(1.01- \\
1.78)\end{array}$ \\
\hline $\begin{array}{l}\text { Karesvuo, } \\
2013\end{array}$ & $\begin{array}{l}\text { NPHS; } \\
\text { Finland } \\
\text { 2000-2001 }\end{array}$ & $\begin{array}{l}\text { Cross- } \\
\text { sectional }\end{array}$ & 1 & $\geq 30$ & $54 / 1751$ & $\begin{array}{c}\text { Alveolar bone } \\
\text { loss }\end{array}$ & $\begin{array}{l}\text { Clinical } \\
\text { diagnosis }\end{array}$ & - & $\begin{array}{c}\mathrm{OR}=1.90 \\
(0.90- \\
4.30)\end{array}$ \\
\hline $\begin{array}{l}\text { Wagley, } \\
2015\end{array}$ & $\begin{array}{c}\text { US } \\
\text { NHAHES } \\
\text { III; US } \\
\text { 1988-1994 }\end{array}$ & $\begin{array}{l}\text { Cross- } \\
\text { sectional }\end{array}$ & 6 & $\geq 40$ & $940 / 8208$ & $\begin{array}{c}>10 \% \text { of sites } \\
\text { with }>3 \mathrm{~mm} \text { of } \\
\text { CAL }\end{array}$ & $\begin{array}{c}\text { Ocular } \\
\text { fundus } \\
\text { photographs }\end{array}$ & $\begin{array}{c}\text { Age } \leq 60 \\
\text { Age }>60 \\
\text { All }^{\&}\end{array}$ & $\begin{array}{c}\mathrm{OR}=1.96 \\
(1.22- \\
3.14) \\
1.32(0.93- \\
1.90) \\
1.36(0.98- \\
1.90)\end{array}$ \\
\hline Shin, 2017 & $\begin{array}{c}\text { KNHANES; } \\
\text { Korea } \\
2008-2010 \\
\text { and } 2012\end{array}$ & $\begin{array}{l}\text { Cross- } \\
\text { sectional }\end{array}$ & 2 & $\geq 40$ & $732 / 13072$ & $\begin{array}{c}\text { CP index } \\
\text { scores } 3 \text { and } 4\end{array}$ & $\begin{array}{c}\text { Ocular } \\
\text { fundus } \\
\text { photographs }\end{array}$ & $\begin{array}{c}\text { Age } \leq 62 \\
\text { Age }>62 \\
\text { All }^{\&}\end{array}$ & $\begin{array}{c}\mathrm{OR}=1.21 \\
(0.90- \\
1.63) \\
0.91(0.74- \\
1.12) \\
1.03(0.86- \\
1.22)\end{array}$ \\
\hline Sun, 2019 & $\begin{array}{l}\text { TNHIRD; } \\
\text { China } \\
\text { 2000-2012 }\end{array}$ & $\begin{array}{l}\text { Retrospective } \\
\text { cohort }\end{array}$ & 13 & $\geq 50$ & $3014 / 83322$ & ICD-9-CM & ICD-9-CM & $\begin{array}{c}\text { Age } \leq 64 \\
\text { Age } \geq 65 \\
\text { All }^{\&}\end{array}$ & $\begin{array}{c}\mathrm{HR}=1.48 \\
(1.34- \\
1.64) \\
1.76(1.57- \\
1.97) \\
1.58(1.46- \\
1.70)\end{array}$ \\
\hline
\end{tabular}

MESA: Multi-Ethnic Study of Atherosclerosis; NPHS: National Population Health Survey 2000; NHAHES: National Health and Nutrition Examination Survey; KNHANES: Korea National Health and Nutrition Examination Survey; TNHIRD: Taiwan National Health Insurance Research Database; ICD: International Classification of Diseases. "All data was calculated by pooling early AMD $P$ and late AMD $P$ using a fixed-effects model for the final analysis; ${ }^{*}$ all data was calculated by pooling different age status $P$ using a fixed-effects model for the final analysis.

TABLE 2: Adjusted variables in studies included in the meta-analysis.

\begin{tabular}{lc}
\hline Author (year) & \multicolumn{1}{c}{ Variables of adjustment } \\
\hline Klein (2008) & Age, gender, race/ethnicity, and study site.
\end{tabular}

(2013)

Wagley (2015)

Shin (2017)

Sun (2019)
Age, smoking, diabetes, carriage of salivary pathogens, and hypertension.

Age, gender, race/ethnicity, education, poverty income ratio, smoking, hypertension, BMI, CRP, and CVD.

Age, gender, education, house income, smoking, hypertension, CVD, anemia, hepatitis B infection, serum HDL level, BMI, serum ferritin level, and white blood cell count.

Age, gender, hypertension, diabetes, hyperlipidemia, asthma/COPD, CLD, and CKD.

Abbreviations: BMI: Body Mass Index; CRP: C-reactive protein; CVD: cardiovascular disease; HDL: high-density lipoprotein; COPD: chronic obstructive pulmonary disease; CLD: chronic liver disease and cirrhosis; CKD: chronic kidney disease. 


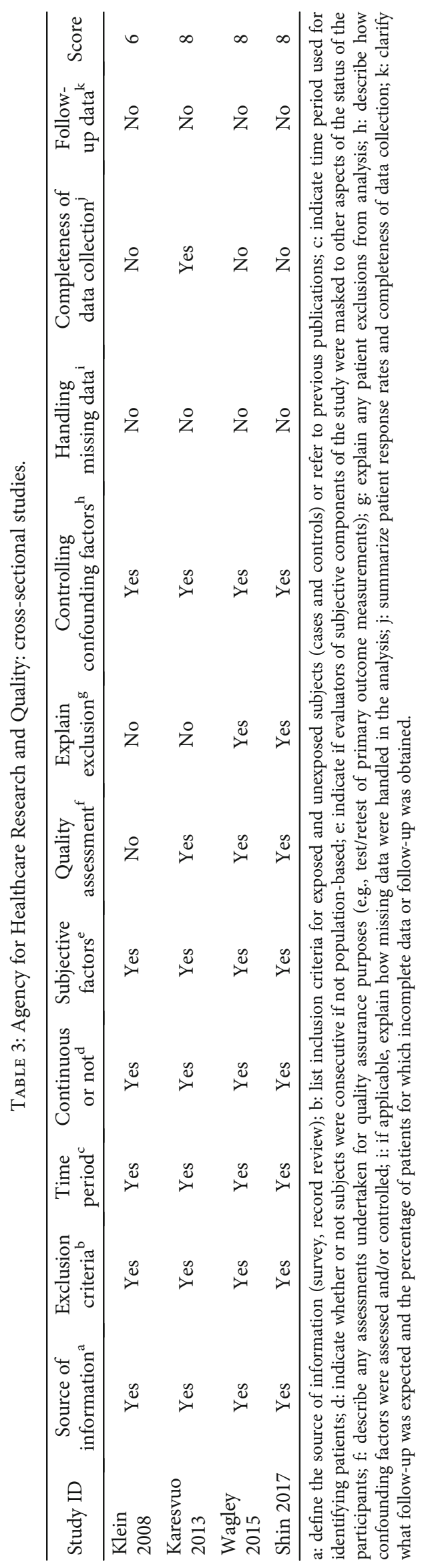




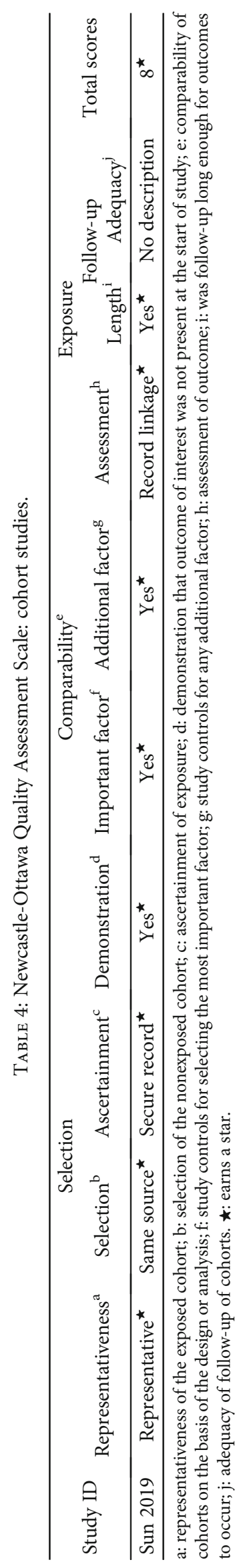




\begin{tabular}{|c|c|c|c|}
\hline \multicolumn{2}{|l|}{$\begin{array}{l}\text { Study } \\
\text { ID }\end{array}$} & $\mathrm{RR}(95 \% \mathrm{CI})$ & $\begin{array}{c}\% \\
\text { Weight }\end{array}$ \\
\hline Klein 2008 & & $1.34(1.01,1.78)$ & 20.53 \\
\hline Karesvuo 2013 & & $1.90(0.90,4.30)$ & 6.70 \\
\hline Wagley 2015 & & $1.36(0.98,1.90)$ & 18.43 \\
\hline Shin 2017 & & $1.03(0.86,1.22)$ & 25.44 \\
\hline Sun 2019 & $\rightarrow$ & $1.58(1.46,1.70)$ & 28.89 \\
\hline Overall $\left(I^{2}=80.4 \%, P=0.000\right)$ & & $1.35(1.07,1.70)$ & 100.00 \\
\hline Note: weights are from random-effects analysis & & & \\
\hline 0.233 & & 4.3 & \\
\hline
\end{tabular}

FIgURE 2: Forest plot of studies.

\begin{tabular}{|c|c|c|c|c|}
\hline Study & \multicolumn{2}{|c|}{ Hazard ratio } & HR & $95 \mathrm{CI}$ \\
\hline \multicolumn{5}{|l|}{ Omitting Klein 2008} \\
\hline \multicolumn{5}{|l|}{ Omitting Karesvuo 2013} \\
\hline \multicolumn{5}{|l|}{ Omitting Wagley 2015} \\
\hline \multicolumn{5}{|l|}{ Omitting Shin 2017} \\
\hline \multicolumn{5}{|l|}{ Omitting Sun 2019} \\
\hline \multirow{2}{*}{\multicolumn{5}{|c|}{ Random-effects model }} \\
\hline & & & & \\
\hline & 0.75 & 1 & & \\
\hline
\end{tabular}

Figure 3: The results of the sensitivity analysis.

Our research has the following advantages. First, this study is the first meta-analysis about whether PD and AMD are related. We retrieved and collected all published studies that met the inclusion criteria, and no publication bias was detected. Second, PD and AMD have some common risk factors; all the studies we included provided the effect sizes after adjusting for related confounding factors. In addition, the quality of the included studies were all medium-/high-quality studies, which made our results more reliable. Finally, this study laid the foundation for further exploration of the relationship between PD and AMD and provided a certain theoretical basis for AMD's mechanism exploration and clinical prevention and treatment.

Our research also has some limitations. First, most of the included studies are cross-sectional studies, which cannot explain the causal relationship between PD and AMD. Second, although most studies were based on scientific examination methods to diagnose $\mathrm{PD}$ and $\mathrm{AMD}$, the diagnostic criteria were different, and some diagnoses were based on patient self-reports, which increased the heterogeneity of our research and reduced credibility. Third, we observed significant heterogeneity in the study. Although the subgroup analysis was used to speculate on the source of the heterogeneity, it still had an impact on the reliability of the research results. Fourth, Egger's test and Begg's test results did not find obvious evidence, but because of the too few studies included, it is difficult to determine whether there is publication bias. Fifth, the adjustment of confounding factors was not perfect. Although each study had carried out meticulous research design and tried to control the confounding factors, there were still unmeasured or unknown confounding factors, which may introduce deviations. And the confounding factors adjusted between different studies were different, which increased the heterogeneity of the study and affected the reliability of the final result. Finally, due to the limited number of studies, we only analyzed the relationship between $\mathrm{PD}$ and $\mathrm{AMD}$ in general and could not further explore the impact of PD on AMD patients at different stages or at different ages and the association between PD at different severity levels and AMD.

With the development of the economy and society, the average lifespan of the population has been prolonged, resulting in population aging. It is the responsibility of oral professionals to reduce the incidence of oral infections and possible 


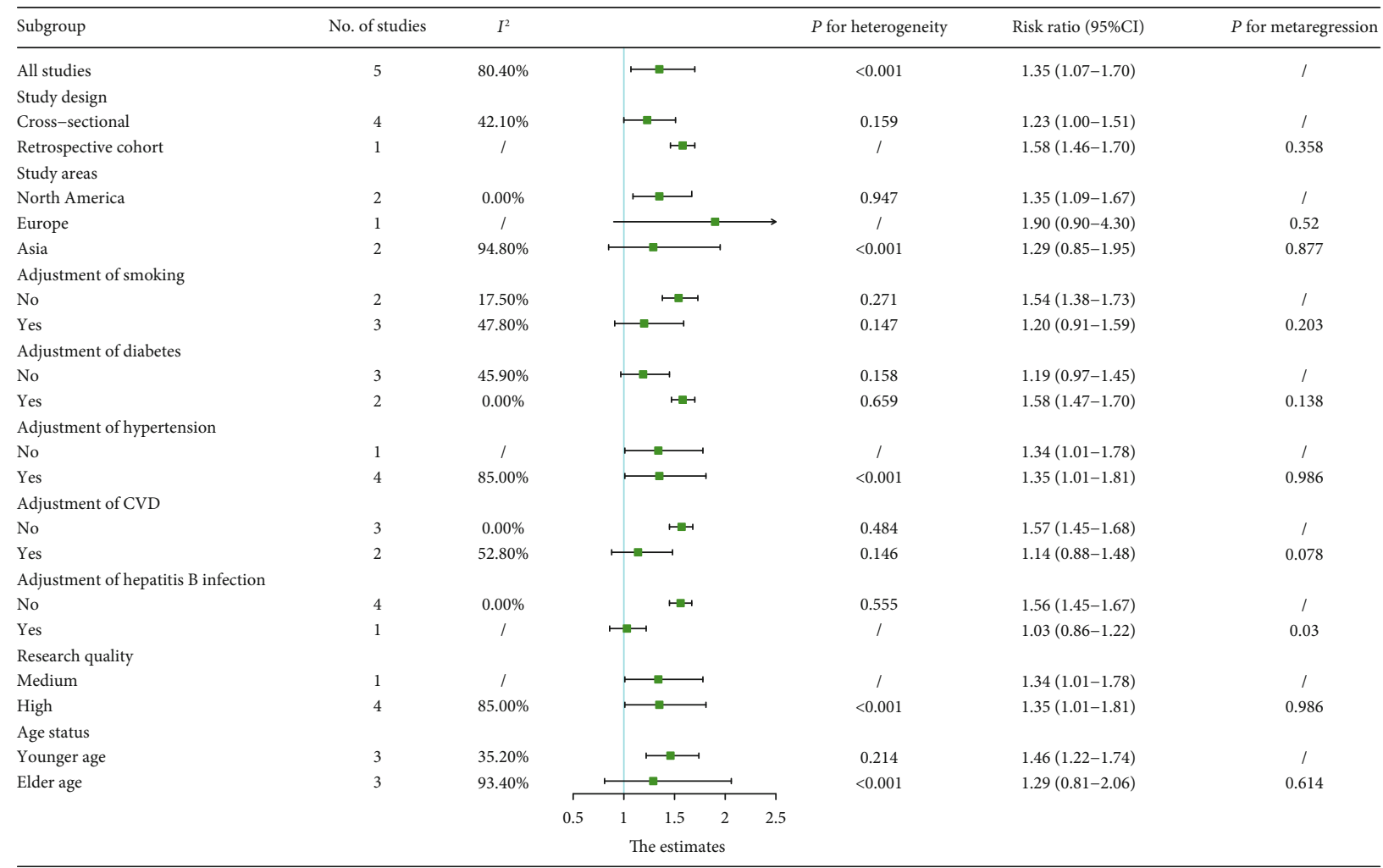

FIgURE 4: The results of the subgroup analysis and metaregression.

systemic diseases caused by PD through early treatment. AMD can cause severe irreversible vision loss in the elderly, which is one of the important causes of blindness. At present, most clinical cases have no effective treatment, which seriously affects the lives of patients. We hope that our research will improve the oral health awareness, help in understanding the importance of maintaining periodontal health, and at the same time lay the foundation for AMD's mechanism exploration and prevention, reduce the risk of AMD, and improve the quality of life in the general population, especially the geriatric population.

\section{Conclusion}

In summary, our meta-analysis of 5 studies showed that $\mathrm{PD}$ is associated with $A M D$, and the risk of AMD in patients with $\mathrm{PD}$ is increased by $35 \%$, but the current evidence is insufficient to confirm the causal relationship between PD and AMD. Researchers need to perform high-quality, large-scale, multi-centre prospective cohort studies and adjust the confounding factors in considerable detail to explore whether these two important diseases are related to each other and the mechanism of their connection.

\section{Conflicts of Interest}

The authors declare that there is no conflict of interest regarding the publication of this paper.

\section{Acknowledgments}

This work was supported by the National Natural Science Foundation of China (No.81500862).

\section{Supplementary Materials}

Appendix 1: electronic search criteria. (Supplementary Materials)

\section{References}

[1] L. S. Lim, P. Mitchell, J. M. Seddon, F. G. Holz, and T. Y. Wong, "Age-related macular degeneration," Lancet, vol. 379, no. 9827, pp. 1728-1738, 2012.

[2] S. Micklisch, Y. Lin, S. Jacob et al., "Age-related macular degeneration associated polymorphism rs10490924 in ARMS2 results in deficiency of a complement activator," Journal of Neuroinflammation, vol. 14, no. 1, 2017.

[3] W. Al-Zamil and S. A. Yassin, "Recent developments in agerelated macular degeneration: a review," Clinical Interventions in Aging, vol. 12, pp. 1313-1330, 2017.

[4] K. Kaarniranta, E. Pawlowska, J. Szczepanska, A. Jablkowska, and J. Blasiak, "Role of mitochondrial DNA damage in ROSmediated pathogenesis of age-related macular degeneration (AMD)," International Journal of Molecular Sciences, vol. 20, no. 10, 2019.

[5] P. Mitchell, G. Liew, B. Gopinath, and T. Y. Wong, "Agerelated macular degeneration,” Lancet, vol. 392, no. 10153, pp. 1147-1159, 2018. 
[6] D. F. Kinane, P. G. Stathopoulou, and P. N. Papapanou, "Periodontal diseases," Nature Reviews. Disease Primers, vol. 3, no. 1, article 17038, 2017.

[7] R. J. Genco and W. S. Borgnakke, "Risk factors for periodontal disease," Periodontol, vol. 62, no. 1, pp. 59-94, 2013.

[8] A. Falcao and P. Bullon, "A review of the influence of periodontal treatment in systemic diseases," Periodontology 2000, vol. 79, no. 1, pp. 117-128, 2019.

[9] D. Bourgeois, C. Inquimbert, L. Ottolenghi, and F. Carrouel, "Periodontal pathogens as risk factors of cardiovascular diseases, diabetes, rheumatoid arthritis, cancer, and chronic obstructive pulmonary disease-is there cause for consideration?," Microorganisms, vol. 7, no. 10, 2019.

[10] L. Rydén, K. Buhlin, E. Ekstrand et al., "Periodontitis increases the risk of a first myocardial infarction: a report from the PAROKRANK study," Circulation, vol. 133, no. 6, pp. 576-583, 2016.

[11] F. D'Aiuto, N. Gkranias, D. Bhowruth et al., "Systemic effects of periodontitis treatment in patients with type 2 diabetes: a 12 month, single-centre, investigator-masked, randomised trial," The Lancet Diabetes and Endocrinology, vol. 6, no. 12, pp. 954-965, 2018.

[12] K. Takeuchi, K. Matsumoto, M. Furuta et al., "Periodontitis is associated with chronic obstructive pulmonary disease," Journal of Dental Research, vol. 98, no. 5, pp. 534-540, 2019.

[13] J. N. Cooke Bailey, M. A. Pericak-Vance, and J. L. Haines, "Genome-wide association studies: getting to pathogenesis, the role of inflammation/complement in age-related macular degeneration," Cold Spring Harbor perspectives in medicine, vol. 4, no. 12, article a017186, 2014.

[14] S. Datta, M. Cano, K. Ebrahimi, L. Wang, and J. T. Handa, "The impact of oxidative stress and inflammation on RPE degeneration in non-neovascular AMD," Progress in Retinal and Eye Research, vol. 60, pp. 201-218, 2017.

[15] V. Behnke, A. Wolf, and T. Langmann, "The role of lymphocytes and phagocytes in age-related macular degeneration (AMD)," Cellular and Molecular Life Sciences, vol. 77, no. 5, pp. 781-788, 2020.

[16] T. E. Van Dyke, "Pro-resolving mediators in the regulation of periodontal disease," Molecular Aspects of Medicine, vol. 58, pp. 21-36, 2017.

[17] G. Hajishengallis, "Periodontitis: from microbial immune subversion to systemic inflammation," Nature Reviews. Immunology, vol. 15, no. 1, pp. 30-44, 2015.

[18] R. Klein, M. D. Knudtson, B. E. K. Klein et al., "Inflammation, complement factor $\mathrm{h}$, and age-related macular degeneration: the Multi-Ethnic Study of Atherosclerosis," Ophthalmology, vol. 115, no. 10, pp. 1742-1749, 2008.

[19] P. Karesvuo, U. K. Gursoy, P. J. Pussinen et al., "Alveolar bone loss associated with age-related macular degeneration in males," Journal of Periodontology, vol. 84, no. 1, pp. 58-67, 2013.

[20] S. Wagley, K. V. Marra, R. A. Salhi et al., "Periodontal disease and age-related macular degeneration: results from the National Health and Nutrition Examination Survey III," Retina, vol. 35, no. 5, pp. 982-988, 2015.

[21] Y. U. Shin, H. W. Lim, E. H. Hong et al., "The association between periodontal disease and age-related macular degeneration in the Korea national health and nutrition examination survey: a cross-sectional observational study," Medicine (Baltimore), vol. 96, no. 14, article e6418, 2017.

[22] K. T. Sun, N. Y. Hsia, S. C. Chen et al., "Risk of age-related macular degeneration in patients with PERIODONTITIS," Retina, 2019.

[23] D. Moher, A. Liberati, J. Tetzlaff, D. G. Altman, and for the PRISMA Group, "Preferred reporting items for systematic reviews and meta-analyses: the PRISMA statement," $B M J$, vol. 339, 2009.

[24] M. E. Wilson, C. C. Dobler, A. S. Morrow et al., "Association of home noninvasive positive pressure ventilation with clinical outcomes in chronic obstructive pulmonary disease: a systematic review and meta-analysis," JAMA, vol. 323, no. 5, pp. 455$465,2020$.

[25] B. Michalska da Rocha, S. Rhodes, E. Vasilopoulou, and P. Hutton, "Loneliness in psychosis: a meta-analytical review," Schizophrenia Bulletin, vol. 44, no. 1, pp. 114-125, 2018.

[26] J. Zhang and K. F. Yu, "What's the relative risk? A method of correcting the odds ratio in cohort studies of common outcomes," JAMA, vol. 280, no. 19, pp. 1690-1691, 1998.

[27] P. E. Ronksley, S. E. Brien, B. J. Turner, K. J. Mukamal, and W. A. Ghali, "Association of alcohol consumption with selected cardiovascular disease outcomes: a systematic review and meta-analysis," BMJ, vol. 342, 2011.

[28] J. Shao, L. Wu, W. D. Leng et al., "Periodontal disease and breast cancer: a meta-analysis of 1,73,162 participants," Frontiers in Oncology, vol. 8, 2018.

[29] J. P. Higgins, S. G. Thompson, J. J. Deeks, and D. G. Altman, "Measuring inconsistency in meta-analyses," BMJ, vol. 327, no. 7414, pp. 557-560, 2003.

[30] C. B. Begg and M. Mazumdar, "Operating characteristics of a rank correlation test for publication bias," Biometrics, vol. 50, no. 4, pp. 1088-1101, 1994.

[31] M. Egger, G. D. Smith, M. Schneider, and C. Minder, "Bias in meta-analysis detected by a simple, graphical test," $B M J$, vol. 315, no. 7109, pp. 629-634, 1997.

[32] S. J. Park, J. H. Lee, S. J. Woo et al., "Age-related macular degeneration," Ophthalmology, vol. 121, no. 9, pp. 17561765, 2014.

[33] A. Lipecz, L. Miller, I. Kovacs et al., "Microvascular contributions to age-related macular degeneration (AMD): from mechanisms of choriocapillaris aging to novel interventions," GeroScience, vol. 41, no. 6, pp. 813-845, 2019.

[34] D. S. W. Ting, C. Y. L. Cheung, G. Lim et al., "Development and validation of a deep learning system for diabetic retinopathy and related eye diseases using retinal images from multiethnic populations with diabetes," JAMA, vol. 318 , no. 22, pp. 2211-2223, 2017.

[35] The Cochrane Collaboration, C. Li, Z. Lv et al., "Periodontal therapy for the management of cardiovascular disease in patients with chronic periodontitis," Cochrane Database of Systematic Reviews, vol. 11, 2011.

[36] The Cochrane Collaboration, Z. Iheozor-Ejiofor, P. Middleton, M. Esposito, and A.-M. Glenny, "Treating periodontal disease for preventing preterm birth in pregnant women," Cochrane Database of Systematic Reviews, vol. 6, 2017.

[37] P. B. Lockhart, A. F. Bolger, P. N. Papapanou et al., "Periodontal disease and atherosclerotic vascular disease: does the evidence support an independent association?: a scientific 
statement from the American Heart Association," Circulation, vol. 125, no. 20, pp. 2520-2544, 2012.

[38] M. V. Kalayoglu, D. Bula, J. Arroyo, E. S. Gragoudas, D. D'Amico, and J. W. Miller, "Identification of Chlamydia pneumoniae within human choroidal neovascular membranes secondary to age-related macular degeneration," Graefe's Archive for Clinical and Experimental Ophthalmology, vol. 243, no. 11, pp. 1080-1090, 2005.

[39] J. Ambati, J. P. Atkinson, and B. D. Gelfand, "Immunology of age-related macular degeneration," Nature Reviews. Immunology, vol. 13, no. 6, pp. 438-451, 2013. 